\title{
Can Spectral Doppler high specificity and Gray scale nail assessment suggest inflammation in Psoriatic Arthritis patients and Control Groups?
}

\begin{abstract}
Introduction: The use of high resolution ultrasonography (US) has become a very important tool in nail assessment. This study evaluated nails clinical and ultrasound measurements in psoriatic arthritis (PsA) patients.

Methods: A cross sectional study was performed with a total of 60 patients, PSA patients, 10 healthy individuals and 6 hand osteoarthritis patients (OA). Only PsA patients meeting the CASPAR criteria were included in the study.

Results: Spectral Doppler (sD) was used to analyze 208 nail beds. Nail plates on the gray scale (GS) showed loss of the normal three-layered pattern in 89 nails $(45.9 \%)$. Power Doppler (PD) signal was detected in 189 nail beds $(92.2 \%)$, showing inflammatory activity in most of the patients and nails assessed. Resistance index (RI) was significantly lower in PsA patients as compared to control groups in both the longitudinal and transverse planes $(\mathrm{p}<0.001)$.Nail enthesitis was observed when RI values were below 0.4 , characterizing $100 \%$ sensitivity and $96 \%$ specificity $(\mathrm{p}<0.01)$.
\end{abstract}

Conclusions: Nail US scanning presented statistical significance in PsA patients. Future studies could show many inflammatory situations, requiring treatment assessment.

Keywords: psoriatic arthritis, nail ultrasound, spectral Doppler, Power Doppler
Volume 13 Issue 6 - 202I

\author{
José Alexandre Mendonça, Vânia Aparecida \\ Leandro-Merhi, José Luis Braga de Aquino \\ PhD, Professor Doctor of the Postgraduate Program in Health \\ Sciences, Puc-Campinas-SP-Brazil
}

\author{
Correspondence: José Alexandre Mendonça, Postgraduate \\ Program in Health Sciences, Puc-Campinas-SP-Brazil \\ Av. John Boyd Dunlop, s/n - Jardim Ipaussurama, Campinas - SP - \\ Brazil, Cep - 13034-685, Tel +55 019 8135-8322, \\ Email mendoncaja.us@gmail.com
}

Received: October 25, 2021 | Published: November 16, 2021
Abbreviations: GS, gray scale; OA, osteoarthritis; PASI, psoriasis area and severity index; PD, power Doppler; PsA, psoriatic arthritis; RA, rheumatoid arthritis; RI, resistance index; ROC, receiver operating curve; sD, spectral Doppler; SD, standard deviation; US, ultrasonography; VASGp, visual analog scale for global pain; VASNp, visual analog scale for nail pain; $\mathrm{mNGS}$, nail measured in gray scale

\section{Introduction}

PsA is a joint inflammatory disease with both peripheral and axial manifestations. It is recognized that enthesitis is an important lesion and a multifaceted skeletal manifestation of this disease. ${ }^{1}$ A few studies have shown enthesopathy in asymptomatic large insertions of the lower limbs in patients with spondyloarthropathies including PsA. ${ }^{2}$ Several studies have demonstrated subclinical enthesopathy or osteitis in as many as $50 \%$ of psoriasis patients with no skeletal symptoms. ${ }^{3}$

Nail plate involvement is a very common psoriasis feature and affects approximately $10 \%$ to $78 \%$ of psoriatic patients. Nail anatomic structure has been recognized as an enthesis which is the target of the inflammatory process in PsA. Different studies have shown that up to $30 \%$ of psoriatic patients have PsA. Out of these, $70 \%-80 \%$ experienced nail changes. ${ }^{4,5}$ A patient with nail psoriasis should be screened for early signs and symptoms of PsA in order to avoid progressive joint damage. ${ }^{6}$

US has been increasingly used to evaluate joint diseases. It has been found to be as good as magnetic resonance imaging in the detection of synovitis. ${ }^{7}$ Advantages of US include noninvasiveness, safety, low cost, lack of radiation exposure, and high patient acceptability; this method enables a rapid high-resolution soft tissue examination and is able to scan multiple joints at one time. High resolution US technology includes GS, PD and sD ultrasound. This technological tool has been used and validated in assessing disease activity in rheumatoid arthritis. ${ }^{8-10} \mathrm{PD}$ and $\mathrm{sD}$ offer the advantage to estimate both high as well as low velocity blood flow quantitatively. $\mathrm{PD}$ to some extent provides a reflection of the degree of angiogenesis, which is critically related to joint damage and therapeutic responses to drugs. ${ }^{11,12}$ Extra-synovial PD signal has been detected in both PsA and psoriasis patients as in entheses and paratendon. ${ }^{13-16}$ So, the RI is a quantitative index that has been used to evaluate tissue abnormal blood flow in inflammatory arthropathy. ${ }^{17}$

The high-resolution US evaluation has become an important tool in PsA joint lesions assessments with diagnostic perspectives. ${ }^{18-20}$ There are many nail findings to be obtained by ultrasound, mainly in relation to $\mathrm{sD}$. A previous study has demonstrated that patients with psoriasis have lower blood flow in the nail fold compared with healthy control group. ${ }^{21}$ In this study we evaluated sD nail findings in PsA patients as compared with healthy individuals and patients with hand OA. Our hypothesis was that sD nail findings could help detect active inflammatory processes in PsA patients.

\section{Methods}

The study was performed at the Instituto de Pesquisa Clínica de Campinas - IPECC and at Pontifícia Universidade Católica of Campinas in both the Rheumatology and Dermatology Divisions. Patients were assessed as routine outpatients at the Rheumatology and Dermatology Divisions and underwent US evaluation as an extension of their physical examination. All participants were instructed about the protocol and informed consent was secured before study procedures. Ethical approval was obtained before starting the study 
protocol (opinion number: 1.526.307). The nail bed assessment scores with $\mathrm{SD}$, semiquantitative GS and PD were not randomly selected because prior standards are not available in the literature.

\section{Patient groups and clinical assessment}

A total of 44 consecutive PsA patients, 10 healthy individuals and 6 patients with hand OA were included. PsA patients were included if they met the CASPAR criteria. ${ }^{22}$ Clinical assessment was performed by a rheumatologist and a dermatologist who were blinded to the ultrasound findings. Patients were clinically assessed before US examination and the following parameters were evaluated in connection with the disease: age, gender, ethnic group, psoriasis area and severity index (PASI), patient's global pain and nail pain (each 0 to $100 \mathrm{~mm}$ in the visual analogue scale for global pain -VASGp and in the visual analogue scale for nail pain VASNp, respectively), and disease and treatment duration. Specific medication use was also recorded for all patients.

The US examinations of the musculoskeletal system were performed with multiplanar techniques, focusing the clinically involved sites, adopting the indications provided by the European League Against Rheumatism guidelines for musculoskeletal ultrasound in rheumatology. ${ }^{23}$

US was performed by a rheumatologist fully trained in musculoskeletal ultrasound and with a special interest in scanning enthesitis. Ultrasound was performed using a MyLab (Esaote S.p.A., São Paulo-Brazil) equipment with a high frequency GS probe
(18MHz). US evaluation was performed with GS and PD techniques to verify joint echotexture damage. The PD specifications have been standardized through a frequency ranging e from 8.0 to $12.5 \mathrm{MHz}$ and pulse repetition frequencies (PRF) ranging from 0.7 to $1.0 \mathrm{kHz}$. In order to quantify the PD signal sD was used, which identifies real blood flow.

Nail abnormalities detected by US were graded with both GS and PD techniques. Nail abnormalities observed in the GS were semiquantitatively graded from 0 to 3 , as follows: GS0-preserved threelayered nail pattern; GS1-mild abnormality in the three-layered nail pattern or one sinuosity or nail loss; GS2-intense abnormality in the three-layered nail pattern, still preserving some segments of the nail, more than one sinuosity or nail loss; and GS3- total loss of the threelayered nail pattern, thickening of all the nail bed with loss of the eco texture or the nail insertion point $(\geq 2.5 \mathrm{~mm}$ ) (Figure 1). The nail bed (distance between the ventral plate and the bone margin of the distal phalanx) was measured in both the longitudinal plane (longitudinal mNGS, $\mathrm{mm}$ ) and the transversal plane (transversal mNGS, mm). Nail PD abnormalities (PD0-3) were graded to measure the presence and intensity of nail enthesitis as follows: PD0: there is no in the nail bed flow; PD1: presence of 1 point or $25 \%$ of the PD signal especially at the insertion point of the nail; PD2: presence of $<50 \%$ of the PD signal especially at the insertion point of the nail; PD3: presence of $>50 \%$ of PD signal especially at the insertion point of the nail (Figure 2). SD was used to measure RI in the nail bed in both the longitudinal plane and the transversal plane (Figure 3).
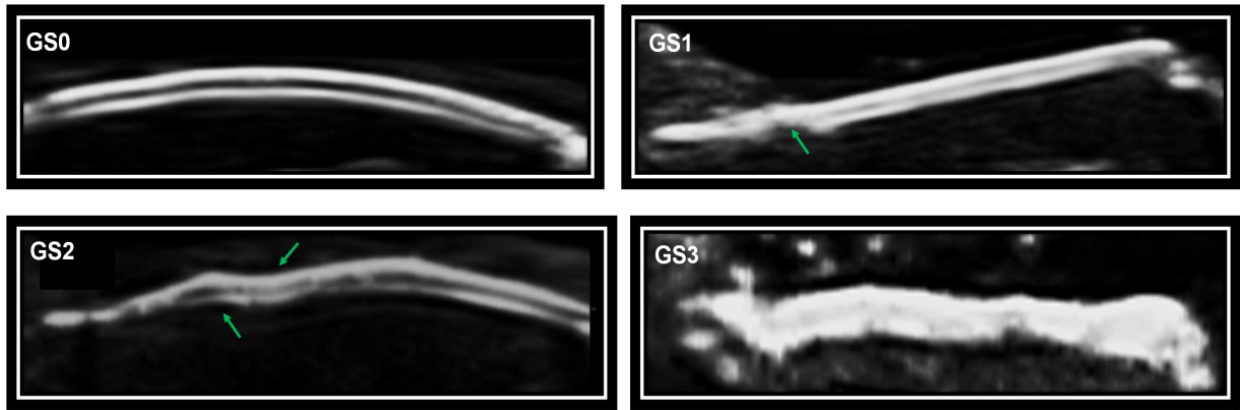

Figure I Semiquantitative GS ultrasound score scale for nail abnormalities.

GS0, preserved three-layered nail pattern; GSI, light abnormality in the three-layered nail pattern or one sinuosity or nail loss; GS2, intense abnormality in the three-layered nail pattern, still preserving some segments of the nail, more than one sinuosity or nail loss; and GS3, Total loss of the three-layered nail pattern, thickening of all of the nail bed with loss of the eco texture or the nail insertion point $(\geq 2.5 \mathrm{~mm})$.
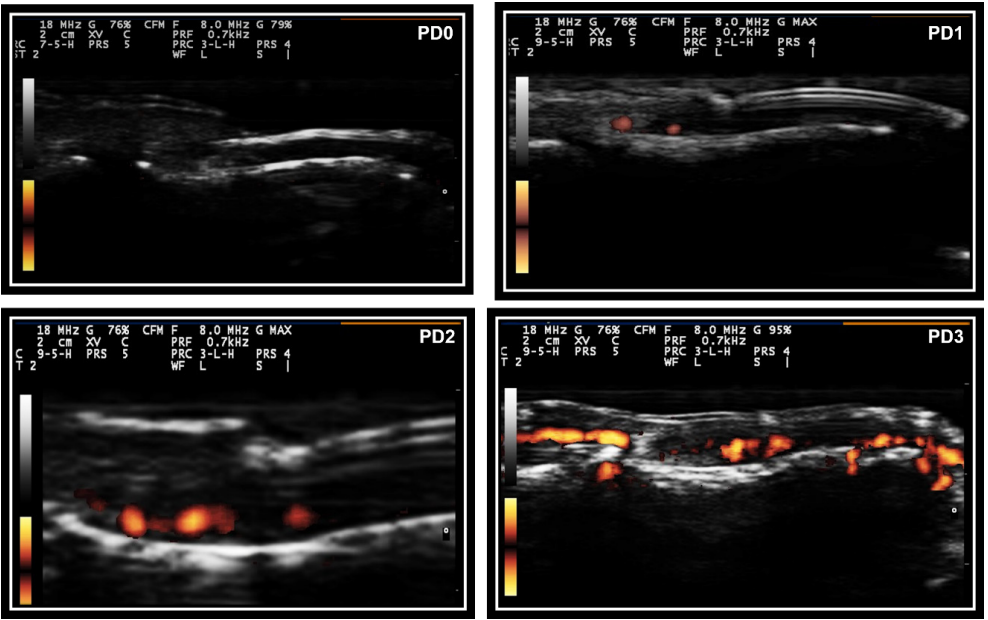

Figure 2 Semiquantitative PD score for nail abnormalities.

Legend- PD0, Signal power Doppler grade 0; PDI, Signal power Doppler grade I; PD2, Signal power Doppler grade 2; PD3, Signal power Doppler grade 3.

Citation: Mendonça JA, Leandro-Merhi VA, Aquino JLB. Can Spectral Doppler high specificity and Gray scale nail assessment suggest inflammation in Psoriatic Arthritis patients and Control Groups? MOJ Orthop Rheumatol. 2021;13(6):I37-I42. DOI: 10.15406/mojor.202I.13.00564 

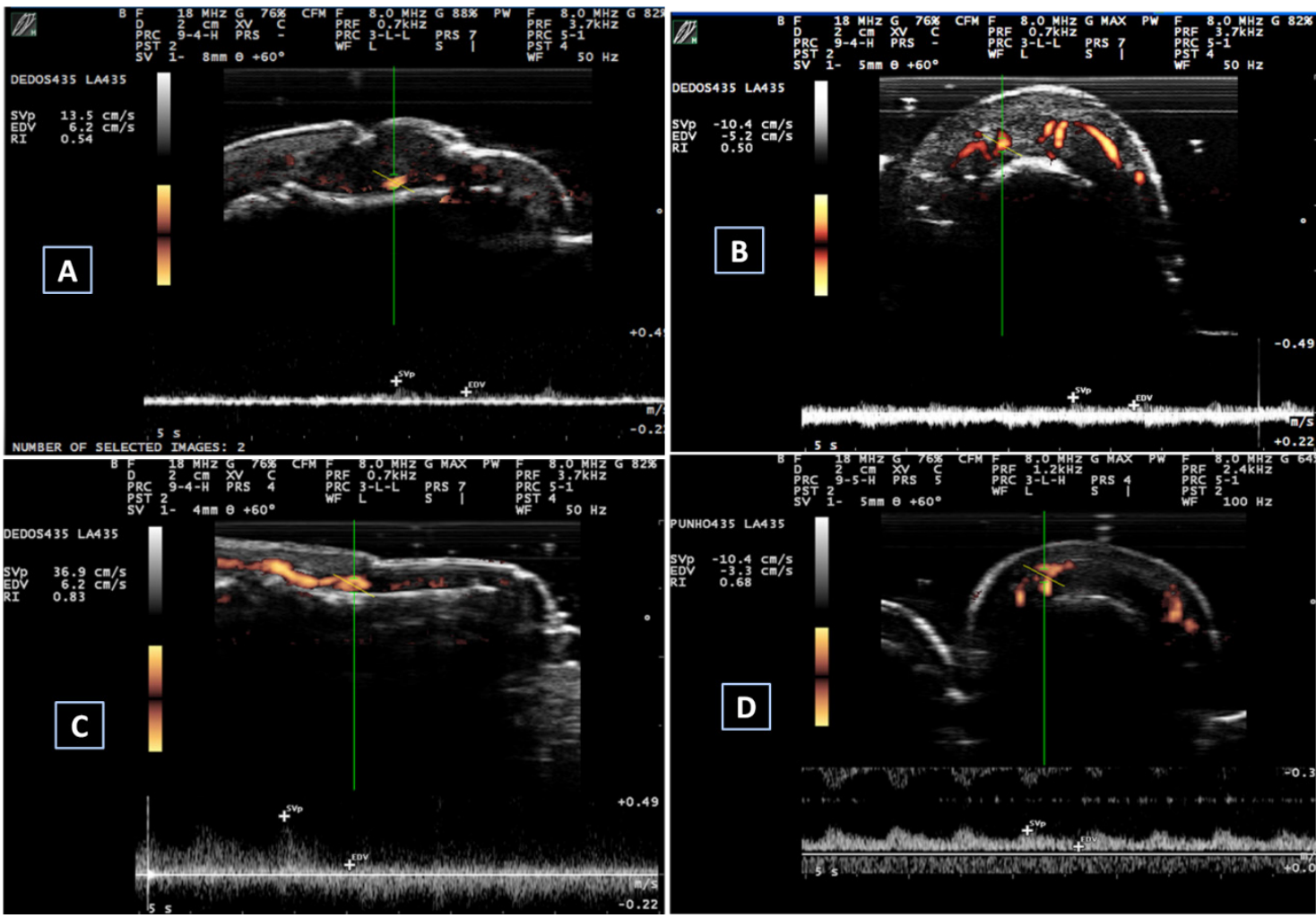

Figure 3 Spectral Doppler measurements.

A and B: spectral Doppler measurements in a PsA patient. (A) Longitudinal spectral Doppler $-\mathrm{RI}=0.54$; (B) Transversal spectral Doppler $-\mathrm{RI}=0.50$; C and D: spectral Doppler measurements in a control individual. $(\mathbf{C})$ Longitudinal spectral Doppler $-\mathrm{RI}=0.83$ and $\mathbf{D}$. Transversal spectral Doppler $-\mathrm{RI}=0.68$.

\section{Statistics}

Data are expressed either as frequencies or means $( \pm$ standard deviation [SD]) according to the variable. The mean values for $\mathrm{sD}, \mathrm{GS}$ and PD parameters in PsA patients and their controls were compared by using a two-tailed $t$ test. The Mann-Whitney $\mathrm{U}$ test was used to compare ultrasound semi quantitative scores between PsA patients and controls. Correlation coefficients between clinical parameters (age, PASI, disease duration, VASGp and VASNp) and sD, GS and PD data were reviewed using the Pearson and Spearman correlation tests.

The performance of $\mathrm{sD}$ measurements to diagnose PsA and clinical parameters in PsA patients was assessed using the ROC curve analysis. Statistical analysis was performed using SPSS V.11.5 and p values lower than 0.05 were considered significant.

\section{Results}

A total of 208 nail beds was assessed using sD. The first, second, third, fourth and fifth nail bed exam revealed abnormalities in 15 (7.2\%), 63 (30.3\%); 61 (29.3\%); 41 (19.7\%); and 28 (13.5\%) nail beds, respectively. Using this technique, a total of 192 longitudinal and 153 transversal RI measurements were performed. The number of longitudinal and transversal measurements of the nail bed thickness was 120 and 90, respectively. Nail appearance on GS showed loss of the normal three-layered pattern (GS2 or GS3) in 89 nails (45.9\%).

VASGp was graded as 0 (no pain) in 31 individuals of our sample $(70.5 \%)$. Accordingly, VASNp or pain in the distal interphalangeal joint was graded as 0 (no pain) in the vast majority of the nails evaluated $(n=186,89.4 \%)$. Demographic data and clinical parameters of PsA patients and their controls evaluated by US in our sample are shown in Table 1. PsA patients were not older than their controls and showed a trend to be more symptomatic for global pain $(\mathrm{p}=0.07)$ while they experienced significantly more nail pain than their healthy controls and patients with hand OA $(\mathrm{p}=0.004)$.

PD signal was detected in 189 nail beds (92.2\%), showing inflammatory activity in most of the patients and nails examined. Results for nail US are shown in Table 2. RI measured by sD was significantly lower in PsA patients as compared to their controls in both longitudinal and transversal planes $(\mathrm{p}<0.001)$. In general, GS or PD nail abnormalities were significantly more prevalent in PsA patients as compared to their controls. Accordingly, longitudinal nail bed measurements were significantly longer in PsA patients as compared with healthy controls and hand OA patients. Transverse measurements did not differ significantly between PsA patients and their controls.

A significant association between clinical parameters and US findings was observed in our sample. Most of the correlations found were weak to moderate. VASGp for example was moderately correlated with longitudinal RI $(\mathrm{r}=0.322 ; \mathrm{p}=0.037)$. As expected, semiquantitative nail PD scores were significantly correlated with the presence of $\mathrm{PD}$ signal $(\mathrm{r}=0.632 ; \mathrm{p}<0.01)$, while semiquantitative nail GS scores were also strongly associated with nail GS ( $\mathrm{r}=0.842$; $\mathrm{p}<0.01)$. Interestingly, mNGS transverse measurements were also strongly associated with GS abnormalities and lack of the normal three-layered nail pattern 
Table I Demographic data and clinical parameters for PsA patients and their controls evaluated by nail US

\begin{tabular}{|c|c|c|c|}
\hline & $\begin{array}{l}\text { PsA } \\
(n=44)\end{array}$ & $\begin{array}{l}\text { Control } \\
(n=16)\end{array}$ & $\mathbf{p}$ \\
\hline Age, years (mean $\pm S D$ ) & $45.27 \pm 14.68$ & $40.87 \pm 19.06$ & 0.45 \\
\hline Sex Male & $28(63.6 \%)$ & $5(3 \mid .3 \%)$ & 0.54 \\
\hline Female & $16(36.4 \%)$ & II (68.8\%) & 0.43 \\
\hline \multicolumn{4}{|l|}{ Ethinicitys } \\
\hline Caucasian & $40(90.9 \%)$ & $13(8 \mid .3 \%)$ & 0.45 \\
\hline Black & $2(4.5 \%)$ & $3(18.8 \%)$ & 0.67 \\
\hline Asian & 2 (4.2\%). & & 0.56 \\
\hline PASI (mean \pm SD) & $4.79 \pm 10.40$ & & \\
\hline Disease duration, months (mean $\pm S D$ ) & $13.04 \pm 21.23$ & & \\
\hline VASGp, cm (mean $\pm S D)$ & $2.02 \pm 3.54$ & $0.37 \pm 1.50$ & 0.07 \\
\hline VASNp, cm (mean \pm SD) & $0.51 \pm 1.71$ & $0.00 \pm 0.00$ & 0.004 \\
\hline Treatment duration, months (mean $\pm S D$ ) & $13.04 \pm 21.42$ & & \\
\hline \multicolumn{4}{|l|}{ Medication } \\
\hline None & $25(56.8 \%)$ & & \\
\hline Methotrexate & $9(20.5 \%)$ & & \\
\hline Adalimumab & $3(6.8 \%)$ & & \\
\hline Etanercept & $3(6.8 \%)$ & & \\
\hline Infliximab & $3(6.8 \%)$ & & \\
\hline Acitretine & I (2.3\%) & & \\
\hline
\end{tabular}

US, ultrasonography; PsA, psoriatic arthritis; PASI, psoriasis area and severity index;VASGp, visual analog scale for global pain;VASNp, visual analog scale for nail pain; SD, standard deviation

Table 2 Nail USG parameters in PsA patients and their controls

\begin{tabular}{|c|c|c|c|}
\hline & $\begin{array}{l}\text { PsA } \\
(n=44)\end{array}$ & $\begin{array}{l}\text { Control } \\
(n=16)\end{array}$ & $\mathbf{p}$ \\
\hline Longitudinal RI (mean \pm SD) & $0.48 \pm 0.11$ & $0.77 \pm 0.30$ & $<0.001$ \\
\hline Transversal RI (mean \pm SD) & $0.46 \pm 0.08$ & $0.66 \pm 0.14$ & $<0.001$ \\
\hline Nail PD (0 to 3) (mean \pm SD) & $2.07 \pm 0.91$ & $1.98 \pm 0.81$ & 0.16 \\
\hline Nail GS (0 to 3) (mean \pm SD) & $1.46 \pm 0.88$ & $0.44 \pm 0.69$ & $<0.001$ \\
\hline Nail PD (0 or I) (mean \pm SD) & $0.92 \pm 0.27$ & $0.66 \pm 0.47$ & 0.026 \\
\hline Nail GS (0 or I) (mean \pm SD) & $0.45 \pm 0.17$ & $0.45 \pm 0.21$ & 0.73 \\
\hline mNGS longitudinal, mm (mean \pm SD) & $1.99 \pm 1.25$ & $1.70 \pm 0.4 \mid$ & 0.02 \\
\hline mNGS transverse, mm (mean \pm SD) & $1.23 \pm 1.24$ & $0.76 \pm 0.17$ & 0.29 \\
\hline
\end{tabular}

US, ultrasonography; PsA, psoriatic arthritis; RI, resistance index; PD, power Doppler; GS, gray scale; SD, Standard deviation; mNGS, nail measured in gray scale

\section{Test performance analysis}

A nail inflammatory process was observed when RI values were below 0.4 , characterizing $100 \%$ sensitivity and $96 \%$ specificity, when the area under the ROC curve was $0.858(\mathrm{p}<0.01)$.

\section{Discussion}

In the present study we have demonstrated that $\mathrm{SD}$ measurements are associated with entheseal abnormalities in PsA patients and may help detect inflammatory activity in the management of these patients. GS ultrasound evaluation may demonstrate abnormalities in the shape of the nail bed, loss of the three-layered nail pattern as well as changes in the longitudinal or transversal nail bed in patients with PsA even before clinical defects develop. Using a composite semiquantitative score of the GS, we were able to show nail plate ultrasonographic abnormalities in PsA patients without clear nail clinical findings. Test performance analysis demonstrated that RI measurements using sD in our sample were significantly associated with PsA diagnosis. Currently, the PD characterizes important indications in the joint inflammatory disease; sD parameters have been associated with inflammatory clinical and US changes in spondyloarthritis, as is reported in the literature. ${ }^{24}$ 
The evaluation of RI allows quantification of the degree of microcirculation vasodilation. Previous studies have defined that at the joints the RI value is usually near 1 in healthy subjects; in case of inflammation this parameter decrease; in synovitis and sacroiliitis the cut-off points are $<0.79$ and $\leq 0.75$, respectively, but still in previous studies $^{25,26}$ a clinically validated cut-off value for nail enthesitis was not available. There is a potential use of $\mathrm{sD}$ to evaluate entheseal involvement in rheumatic diseases, especially when the PD generates questions about inflammatory tissue activity.

Measurements using SD may objectively quantify PD signal in PsA patients, when compared to onychomycosis patients. ${ }^{27}$ Current studies have suggested that nail measurements detected by $\mathrm{SD}$ can be associated to an atherosclerotic process in PsA patients. ${ }^{28}$

In our study RI measurements in PsA patients were significantly lower than those of the control group (healthy controls and OA patients). That was demonstrated in both longitudinal and transversal measurements and is consistent with our previous findings. It is very interesting to note that $\mathrm{sD}$ changes precede clinical manifestation in PsA patients. In our sample a total of 31 (70.5\%) PsA patients and 186 (89.4\%) nails presented sD abnormalities while VASGp and VASNp were 0 , meaning absence of clinical symptom. These findings suggest that sD measurements in PsA patients may help identify patients with subclinical inflammatory activity conditions. A systematic review showed studies that characterize PD and sD measurements, but still no other paper had analyzed completely the cut-off point, defining the correlations between clinical findings, GS, PD and $\mathrm{sD}$ in nail entheses of PsA patients and control groups. ${ }^{29}$

At this point it is also important to point out limitations of the present study. The cross sectional design of our protocol precludes establishing a cause-effect association between $\mathrm{sD}$ findings and PsA pathophysiology. This study was a non-randomized selection of nail beds because of the inexistence of prior references. Further studies including a better description of the $\mathrm{SD}$ findings related to nail enthesitis and synovial damage in distal interphalangeal joints in both PsA and OA patients are needed to consolidate our findings.

\section{Conclusions}

In the present study we have demonstrated that nail ultrasound evaluation is a valuable tool to detect inflammatory activity in patients with PsA. SD measurements quantifying inflammatory damage of the entheseal tissue specifically in the nail bed can be used to identify patients with subclinical disease activity and may help in the management of their conditions. Further studies using a longitudinal design will be needed to evaluate whether the therapeutic management of these subclinical ultrasonographic manifestations are associated with better outcomes in PsA patients.

\section{Acknowledgments}

We are grateful for the support of the Pontifical Catholic University of Campinas-SP-Brazil (Puc-Campinas, SP, Brazil).

\section{Conflicts of interest}

The authors declare there are no conflict of interests.

\section{Declaration}

\section{Ethical approval}

This study was approved by the institution's ethics committee (opinion number: 1.526.307).

\section{Funding}

This project was not sponsored.

\section{Statement of authorship/Authors' contributions}

JAM was responsible for the conception and design of the research, acquisition and analysis of the data, and wrote the manuscript. VALM and JLBA equally contributed to the data interpretation; and the critical analysis of the article. All authors revised the manuscript, and are fully accountable for ensuring the integrity and accuracy of the work. The authors read and approved the final manuscript.

\section{References}

1. McGonagle D, Conaghan PG, Emery P. Psoriatic arthritis: a unified concept twenty years on. Arthritis and rheumatism. 1999;42(6):1080 1086

2. Balint PV, Kane D, Wilson $\mathrm{H}$, et al. Ultrasonography of entheseal insertions in the lower limb in spondyloarthropathy. Annals of the rheumatic disease. 2002;61(10):905-910.

3. McGonagle D, Ash Z, Dickie L, et al. The early phase of psoriatic arthritis Annals of the Rheumatic Diseases. 2011;70 Suppl 1:i71-76.

4. Leung YY, Tam LS, Kun EW, et al. Psoriatic arthritis as a distinct disease entity. Journal of Postgraduate Medicine. 2007;53(1):63-71.

5. Lawry M. Biological therapy and nail psoriasis. Dermatologic Therapy. 2007;20(1):60-67.

6. McGonagle D, Tan AL, Benjamin M. The biomechanical link between skin and joint disease in psoriasis and psoriatic arthritis: what every dermatologist needs to know. Annals of the rheumatic diseases. 2008;67(1):1-4.

7. Terslev L, Torp-Pedersen S, Savnik A, et al. Doppler ultrasound and magnetic resonance imaging of synovial inflammation of the hand in rheumatoid arthritis: a comparative study. Arthritis and Rheumatism. 2003;48(9):2434-2441

8. Varsamidis K, Varsamidou E, Tjetjis V, et al. Doppler sonography in assessing disease activity in rheumatoid arthritis. Ultrasound in Medicine \& Biology. 2005;31(6):739-743.

9. Rubin JM, Adler RS, Fowlkes JB, et al. Fractional moving blood volume: estimation with power Doppler US. Radiology. 1995;197(1):183-190.

10. Stevenson GN, Collins SL, Welsh AW, et al. A Technique for the Estimation of Fractional Moving Blood Volume by Using Three-dimensional Power Doppler US. Radiology 2014.

11. Naredo E, Moller I, Cruz A, et al. Power Doppler ultrasonographic monitoring of response to anti-tumor necrosis factor therapy in patients with rheumatoid arthritis. Arthritis and Rheumatism. 2008;58(8):22482256.

12. Hammer HB, Kvien TK. Ultrasonography shows significant improvement in wrist and ankle tenosynovitis in rheumatoid arthritis patients treated with adalimumab. Scandinavian Journal of Rheumatology. 2011;40(3):178-182.

13. Fournie B, Margarit-Coll N, Champetier de Ribes TL, et al. Extrasynovial ultrasound abnormalities in the psoriatic finger. Prospective comparative power-doppler study versus rheumatoid arthritis. Joint, bone, spine: revue du rhumatisme. 2006;73(5):527-531.

14. Tang Y, Cheng S, Yang Y, et al. Ultrasound assessment in psoriatic arthritis (PsA) and psoriasis vulgaris (non-PsA): which sites are most commonly involved and what features are more important in PsA? Quant Imaging Med Surg. 2020;10(1):86-95 
15. Gutierrez M, Filippucci E, Salaffi F, et al. Differential diagnosis between rheumatoid arthritis and psoriatic arthritis: the value of ultrasound findings at metacarpophalangeal joints level. Annals of the Rheumatic Diseases. 2011;70(6):1111-1114

16. Ash ZR, Tinazzi I, Gallego CC, et al. Psoriasis patients with nail disease have a greater magnitude of underlying systemic subclinical enthesopathy than those with normal nails. Ann Rheum Dis. 2012;71(4):553-556.

17. Mendonça JA, Pansani LN, Mimoto MB, et al. Nail enthesis ultrasound and automated software-guided assessment of bilateral common carotid intima-media thickness in psoriasis and psoriatic arthritis: is there a correlation with clinical and laboratory findings? Drugs in Context. 2020;9: 1-2.

18. Ory PA, Gladman DD, Mease PJ. Psoriatic arthritis and imaging. Ann Rheum Dis. 2005;64 Suppl 2:ii55-57.

19. van der Heijde D, Sharp J, Wassenberg S, et al. Psoriatic arthritis imaging: a review of scoring methods. Ann Rheum Dis. 2005;64 Suppl 2:ii61-64.

20. Aydin SZ, Ash ZR, Tinazzi I, et al. The link between enthesitis and arthritis in psoriatic arthritis: a switch to a vascular phenotype at insertions may play a role in arthritis development. Ann Rheum Dis. 2013;72(6):992-995.

21. Husein El-Ahmed H, Garrido-Pareja F, Ruiz-Carrascosa JC, et al. Vessel resistance to blood flow in the nailfold in patients with psoriasis: a prospective case-control echo Doppler-based study. The British Journal of Dermatology. 2012;166(1):54-58.

22. Taylor W, Gladman D, Helliwell P, et al. Classification criteria for psoriatic arthritis: development of new criteria from a large international study. Arthritis and Rheum. 2006;54(8):2665-2673.
23. Backhaus M, Burmester GR, Gerber T, et al. Guidelines for musculoskeletal ultrasound in rheumatology. Annals of the Rheumatic Diseases. 2001;60(7):641-649.

24. Mendonça JA, Bisetto de Andrade B, Braga de Aquino JL, et al. Spectral Doppler and automated software-guided ultrasound assessment of bilateral common carotid intima-media thickness in spondyloarthritis: is there a correlation with clinical findings? Drugs in Context. 2018;7:212538.

25. Carotti M, Salaffi F, Morbiducci J, et al. Colour Doppler ultrasonography evaluation of vascularization in the wrist and finger joints in rheumatoid arthritis patients and healthy subjects. European Journal of Radiology. 2012;81(8):1834-1838

26. Castillo-Gallego C, De Miguel E, García-Arias M, et al. Color Doppler and spectral Doppler ultrasound detection of active sacroiliitis in spondyloarthritis compared to physical examination as gold standard. Rheumatol Int. 2017;37(12):2043-2047.

27. Mendonca JA. Differences of spectral Doppler in psoriatic arthritis and onychomycosis. Revista Brasileira de Reumatologia. 2014;54(6):490 493.

28. Mendonça JA, Pansani LN, Mimoto MB, et al. Nail enthesis ultrasound and automated software-guided assessment of bilateral common carotid intima-media thickness in psoriasis and psoriatic arthritis: is there a correlation with clinical and laboratory findings?. Drugs Context. 2020;9:2020-1-2.

29. Mendonça JA, Aydin SZ, D’Agostino M. The use of ultrasonography in the diagnosis of nail disease among patients with psoriasis and psoriatic arthritis: a systematic review. Adv Rheumatol. 2019;59(1):41. 